\title{
HACIA EL HORIZONTE COMPRENSIVO DE LA CORPOREIDAD: UNA TRANSICIÓN ENTRE LOS ENFOQUES DE HUSSERL Y HEIDEGGER
}

\author{
Luis Fernando ButiérReZ \\ https://orcid.org/0000-0001-9502-2234 \\ Universidad Nacional de La Plata \\ bttp://dx.doi.org/10.15304/ag.39.2.5977
}

\section{Resumen}

En el presente artículo proponemos un abordaje de los análisis en torno al cuerpo en trabajos fundamentales de Husserl y Heidegger, a partir de una distinción de diferencias y continuidades. Buscaremos demostrar que las especificaciones de la corporeidad de Heidegger remiten a una aclaración del entramado comprensivo y de significación en el que también se hallan articulados los análisis fenomenológicos de su maestro. Para ello, en primer lugar, especificaremos las consideraciones de Husserl sobre la corporalidad y la inter-corporalidad, distinguiendo su perspectiva gnoseológica y ética. Luego, dedicaremos un apartado a las aclaraciones de Heidegger en la década de 1920 y otro, a sus descripciones específicas en sus seminarios de la década de 1960, donde desarrolla el carácter ontológico de la corporeidad. Finalmente, analizaremos algunas implicancias relativas a estas tematizaciones, distinguiendo sus enfoques y orientaciones comprensivas sobre el cuerpo.

Palabras clave: corporalidad, comprensión, transición, Husserl, Heidegger.

\begin{abstract}
In the present article we propose an approach of the analyses around the body in fundamental works of Husserl and Heidegger, from a distinction of differences and continuities. We will try to demonstrate that the specifications of Heidegger's corporeality refer to a clarification of the comprehensive and meaningful framework in which the phenomenological
\end{abstract}

Recibido: 15/04/2019. Aceptado: 11/06/2019. 
analyses of his teacher are also articulated. For this, first, we will specify Husserl's considerations on corporality and inter-corporality, distinguishing his gnoseological and ethical perspective. Then, we will devote a section to Heidegger's explanations in the 1920s and another, to his specific descriptions in his seminars of the 1960s, where he develops the ontological character of corporeality. Finally, we will analyze some implications related to these thematizations, distinguishing their comprehensive approaches and orientations about the body.

Keywords: corporality, understanding, transition, Husserl, Heidegger.

\section{Introducción}

En el campo de las interpretaciones de las cuestiones relativas a la corporeidad en la obra de Heidegger, podemos encontrar lecturas y análisis que abordan su escasa tematización en Sein und Zeit o el carácter precursor de sus descripciones en la década de 1960 para las perspectivas relativas a la corporalidad en recepciones posteriores. En tal contexto, con frecuencia distinguimos una lectura progresiva/superadora del enfoque de Heidegger respecto de las respectivas elaboraciones de Husserl. Sin embargo, investigaciones recientes reconocen también continuidades entre estos enfoques.

En el presente artículo proponemos un abordaje de los análisis en torno al cuerpo en trabajos fundamentales de ambos pensadores, a partir de una distinción de diferencias y continuidades. A nuestro entender, las especificaciones de la corporeidad de Heidegger remiten a una aclaración del entramado comprensivo y de significación en el que también se hallan articulados los análisis fenomenológicos de su maestro. En este sentido, sostenemos que la transición entre ambos se debe más bien a un cambio de enfoque que permite desplegar la comprensión del Leib subyacente.

Tal y como Heidegger insiste en sus trabajos respectivos, la pertinencia en el abordaje de cuestiones relativas al cuerpo, la praxis y la ética, por ejemplo, requieren un despliegue comprensivo y especificación metódica que sean correlativos a un desplazamiento respecto de las limitaciones tradicionales. Ello implica un recorrido ineludible por la propia herencia conceptual y semántica de la metafísica para poder establecer las condiciones de una adecuada transición.

Para dar cuenta de ello desplegaremos sus respectivas elaboraciones en obras que entendemos fundamentales: por un lado, Ideen II y Cartesianische Meditationen, de Husserl; por otro, Sein und Zeit y Zollikon Seminare, de Heidegger. Nuestro objetivo consiste en dar cuenta de las consideraciones de lo corporal, poniendo énfasis en sus enfoques y bases comprensivas. 
En primer lugar, especificaremos las consideraciones de Husserl sobre la corporalidad y la inter-corporalidad, distinguiendo su perspectiva gnoseológica y ética. Luego, dedicaremos un apartado a las aclaraciones breves de Heidegger en la década de 1920 y otro, a sus descripciones específicas en sus seminarios de la década de 1960, donde desarrolla el carácter ontológico de la corporeidad. Finalmente, analizaremos algunas implicancias relativas a estas tematizaciones.

De esta manera, proponemos un recorrido por el tratamiento de la corporeidad en la obra de Heidegger y sus posibles contrastes con los desarrollos de Husserl, ambos sustentados en una orientación comprensiva que permite dar cuenta de cruces, confluencias y resonancias entre sus caminos del pensar.

\section{Husserl y sus elaboraciones fenomenológicas de la corporalidad}

Encontramos dos desarrollos fundamentales en torno a la corporalidad en la fenomenología de Husserl: uno en Ideen II y otra en Cartesianische Meditationen (MC). Allí, la metodología fenomenológica parte de la experiencia del cuerpo propio, para pasar luego a la experiencia del cuerpo ajeno. Pero esta aparente dualidad no es tajante: en ambos análisis, Husserl logra una vía de acceso al cuerpo del otro por medio del cuerpo propio. Como veremos, esta perspectiva encuentra huellas de alteridad en el ámbito de la propiedad. Una suerte de doblez o pliegue que, como cinta de Moebius, conduce en su recorrido al otro, apartándose así de lecturas parciales de esta dinámica, tal y como podemos encontrar en ciertas discusiones en su recepción contemporánea ${ }^{1}$.

En Ideen II, Husserl desarrolla un abordaje que vincula conciencia-cuerpo-mundo por varias vías, entre las cuales se destaca el paso del yo puro y neutro al yo personal, con su carácter corpóreo, histórico y situacional. Allí, las relaciones comunitarias e intersubjetivas se muestran entrelazadas, de un modo contrapuesto al aparente carácter aislado y general del ego puro del primer tiempo de la epojé. De esta manera, el mundo se manifiesta como un ámbito co-constituido y compartido, en una trama común de sentidos.

${ }^{1}$ Puede sostenerse que el enfoque de Husserl supera la dicotomía clásica externalismointernalismo. Al respecto, véase Adrián, "La actualidad de la fenomenología husserliana: superación de viejos tópicos y apertura de nuevos campos de exploración”, Eidos N.18 (2013), pp. 25ss. 
La distinción fundamental de la que parte en ambas obras remite a las manifestaciones del cuerpo en su ámbito originario: es un cuerpo físico como cualquier otro (Körper), pero al mismo tiempo es un cuerpo orgánico (Leib) sentido y vivido desde dentro (CM, pp. 127-9 [159-61])². Así, entre los cuerpos físicos del estrato unitario del ego trascendental se destaca primariamente mi cuerpo físico-orgánico, al cual comando de modo inmediato y desde cuyas sensaciones experimento la naturaleza y la propia corporalidad orgánica. Asimismo, la posición del propio cuerpo determina una perspectiva en el mundo desde la cual se da la percepción de los demás cuerpos.

A continuación, desarrollaremos estas dos elaboraciones con sus énfasis respectivos: en Ideen II, analizaremos sus consideraciones de la vivencia (Erlebnis) del cuerpo vivo propio, que permiten plantear una orientación ética de la corporeidad; en $M C$, desarrollaremos la manifestación del otro en su carácter extraño a través de su cuerpo vivo, en una vertiente eminentemente gnoseológica. Como veremos, ambas orientaciones requieren una serie de presupuestos para sortear las limitaciones propias de su enfoque.

\section{1.a. Del sujeto encarnado a la intercorporalidad}

La fenomenología estática analiza situaciones en el desarrollo de la vida del yo; por su parte, la fenomenología genética tiene en cuenta las remisiones retrospectivas de la situación temporal en la que se encuentra el yo, complementando el enfoque anterior. Los primeros trabajos de Husserl corresponden al primer enfoque y sus trabajos de madurez, al último. En este marco, las descripciones en Ideen II se enfocan en la esfera del ego primordial, atendiendo su constitución y despliegue. Ello permite poner en evidencia las constituciones intersubjetivas y los nexos con los otros, en su dimensión histórica y temporal en general.

Ello se debe a que los tratamientos sobre la corporalidad y sus procesos de constitución en dicha obra fueron inicialmente redactados en 1912 y finalmente publicados en 1952, lo cual supone la inclusión de un conjunto de reelaboraciones ${ }^{3}$. Debido a tales agregados, no es posible plantear una progresión lineal de la temática de la corporalidad, pues posiblemente Hus-

${ }^{2}$ Las citas de las obras de Husserl y de Heidegger indican en primer lugar la respectiva abreviatura, luego la paginación del texto original y, entre paréntesis, la traducción utilizada, cuya mención específica realizaremos en la bibliografía.

${ }^{3}$ Véanse las observaciones respectivas, en Sawicki, "Body, Text and Science: the Literacy of Investigative Practices and the Phenomenology of Edith Stein”, in Stein's work for Husserl, Dordrecht: Kluwer (1997), pp. 158-64. 
serl fue cambiando perspectivas y enfoques, en sintonía con los resultados de sus investigaciones ${ }^{4}$. Esta obra y sus vaivenes se distinguen de aquellos desarrollos de la conciencia absoluta y trascendental en el primer tomo de Ideen, trabajo más bien abocado a la superación del dualismo cartesiano entre sustancia pensante y extensa (y su enfoque circunscripto a los aspectos cósicos/mecánicos del cuerpo $)^{5}$.

Específicamente, en Ideen II encontramos desarrollos fundamentales de la corporalidad a partir del parágrafo 35 de la segunda sección, donde Husserl despliega un análisis de la realidad natural del hombre desde los vínculos entre la vida anímica y corporal, revelando las imbricaciones en el mundo humano compartido. Partiendo del yo puro en "su" corriente de vivencias, distingue tres estratos o realidades: material, anímica-animal y espiritual ${ }^{6}$.

En este marco, la mención de alma (Seele) remite a la vinculación entre cuerpo y vida psíquica e incumbe al carácter animal del ser humano y, por supuesto, a la vida animal no humana ${ }^{7}$. Tal imbricación es la vía para apartarse del carácter neutro o general del ego puro y enfocarse en el aspecto mundanal y corpóreo: la comprensión de la irreductibilidad cuerpo-ego y del carácter temporal e histórico de la persona en el mundo se pone aquí de manifiesto.

Comenzando su análisis por el cuerpo orgánico, vivo y propio, Husserl distingue el carácter vinculante entre la realidad espiritual y material. A diferencia del cuerpo físico, relativo al espacio y a la realidad natural o animal del hombre, el cuerpo propio comparte elementos de aquellos tres estratos. Así, la comprensión del cuerpo como expresión espiritual y anímica tiene implicancias correlativas que van de un enfoque natural-material y perceptivo, hasta otro personalista que atiende las manifestaciones culturales, axiológicas y comunitarias, dando cuenta del mundo circundante (Umwelt) en su entramado interrelacional. El medio para ello es el análisis fenomenológico de la experiencia de la mano, la vista, el oído y la vinculación cuerpo-voluntad.

${ }^{4}$ Aquí seguimos la interpretación de Inverso, El mundo entre paréntesis. Una arqueología de las nociones de reducción y corporalidad, Buenos Aires, Prometeo, 2014, p. 78.

${ }^{5}$ El enfoque estático de este primer tomo apunta a desarrollar una fenomenología pura desde una provisional aniquilación del mundo de las cosas, quedando como irreductible la manifestación de una conciencia sin cuerpo. En el marco de estas distinciones, podemos encontrar un breve desarrollo sobre la naturaleza de las realidades materiales y el ser corporal (unidad en donde se enlaza conciencia y cuerpo), como base para la relación cognoscente con el entorno y con los demás, en Husserl Ideen I, pp. 104ss. [125-131].

${ }^{6}$ Véase Husserl Ideen II, pp. 109s. [145ss.]; 137-43 [175-82].

${ }^{7}$ Véase Ibíd., pp. 157 [196s.]. 


\section{1.b. Desde el tocar}

En efecto, Husserl desarrolla el análisis de lo que denomina ubiestesias $(E m p f i n d n i s s)^{8}$, en referencia a las sensaciones que porta el cuerpo y se localizan en determinadas zonas del mismo. Estas permiten experienciar el cuerpo desde él, en una doblez que lo distingue de la manifestación meramente material.

En tal sentido, destaca dos experiencias de la percepción táctil. Por un lado, por ejemplo, distingue las sensaciones que aún resuenan cuando el objeto tocado y la mano se separan. Este carácter material-anímico (sensible) del cuerpo (Leibkörper) se manifiesta en diversas relaciones con el mundo entorno. Por otro, subraya la vivencia de sí como cuerpo: toco y me experimento corpóreo en el tocar. Esta experiencia de sí mismo permite articular las dos dimensiones de la corporalidad: evidencian el cuerpo vivo que experimenta el mundo y su propio carácter corpóreo ${ }^{9}$.

Esta correlatividad perceptiva supone el carácter irreductible de la corporalidad en la experiencia: frente a la tradición cartesiana, subraya que no es posible comprender una experiencia sin cuerpo orgánico y físico. En este marco, el estrato primario del cuerpo en el mundo abre una espacialidad y posibilidad de orientación desde la cual se dan las relaciones con los objetos en el mundo circundante. Ello supone cierto perspectivismo en la experiencia, cuya modalidad de manifestación varía según la posición y orientación en el espacio ${ }^{10}$.

Asimismo analiza las relaciones entre cuerpo y voluntad, donde cabe la posibilidad del movimiento espontáneo. Esta potencialidad ligada al movimiento también influye en la modalidad de manifestación de los objetos en la experiencia, es decir, no solo imprime variabilidad en las perspectivas, sino que también permite dar cuenta del carácter transformador del cuerpo vivo en el mundo circundante, donde el sujeto puede comprenderse como contramiembro de la naturaleza material (Ideen II, pp. 152 [191s.]). En tal sentido, Husserl vincula a la expresión yo puedo esta capacidad operatoria y creativa del cuerpo para operar desde y en el mundo, desplegando un con-

${ }^{8}$ Aquí seguimos este neologismo a partir de la traducción de Antonio Zirión. El término utilizado por Husserl es sinónimo de Empfindung (sensación).Véase Husserl Ideen II, pp. 144-7 [184-7].

${ }^{9}$ Desde aquí pueden seguirse las continuidades de estos análisis en trabajos de Derrida y Nancy. Por ejemplo, en Derrida, El tocar, Jean-Luc Nancy, Buenos Aires, Amorrortu, 2011, pp. 31-196.

${ }^{10}$ Véase Husserl Ideen II, pp. 158s. [197s.]. 
junto de consideraciones sobre aspectos éticos, desiderativos y relativos a la libertad de la realidad espiritual del hombre en tanto persona ${ }^{11}$.

Sin embargo, este lugar eminente del cuerpo propio en la experiencia en el mundo circundante también incluye limitaciones. Entre ellas, Husserl destaca la constitución imperfecta del cuerpo con sus aristas que estorban, acortan o limitan la perspectiva. Es decir, la propia constitución material del cuerpo también obstaculiza la perspectiva de sí, cancelando con ello la posibilidad de una accesibilidad completa ${ }^{12}$.

Por otra parte, aquellas manifestaciones del cuerpo permiten articularse con objetividades superiores, es decir, con valores más allá de lo meramente material, orientados a un horizonte ético. Específicamente, distingue sentimientos "sensibles" que remiten a experiencias en el cuerpo que lo desbordan o incomodan, los cuales son correlativos con actos de valoración. Considerados como la base material y corpórea de la vida volitiva y desiderativa (Ideen II, p. 153 [192]), nuestro autor resalta la ligazón corporal de los valores espirituales en la experiencia de la persona, así como también el carácter dinámico de las relaciones con la materialidad fáctica: esta puede ser trascendida o superada, más allá de los valores sensibles dependientes de la afección pasiva, pues permiten trascender las contingencias corporales (muerte, enfermedad, etc.), por ejemplo, por la vía de la pertenencia a una comunidad ética ${ }^{13}$. De esta manera, como instrumento de la voluntad, las vinculaciones entre cuerpo y ética tienen aquí importantes rudimentos: el cuerpo es considerado como posible trampolín para los valores espirituales ${ }^{14}$.

\section{1.c. Hacia la corporalidad del otro}

En esta obra, Husserl también examina el darse de los otros desde su corporalidad. El tratamiento que realiza tiene como horizonte el revelamiento de la naturaleza objetiva en el marco de la manifestación del mundo compartido intersubjetivamente. En lo que respecta al cuerpo material, indaga en la espacialidad y posicionalidad de los cuerpos, distinguiendo entre la protopresencia (en referencia a las vivencias del objeto dado en una

${ }^{11}$ Véase Ibíd, pp. $257-75$ [304-23].

12 Véase Ibíd, pp. 159 [199].

${ }^{13}$ Encontramos un fructífero análisis detallado e interpretación de la corporalidad ética implicada en la trasposición del sentido de Leib en ámbitos relacionales de orden superior al personal, en Rabanaque, "La corporalidad política como corporalidad ética”, Revista pensamiento político N.7 (2016), pp. 93-108.

${ }^{14}$ Para un análisis de la significación ética del cuerpo propio en Husserl y sus derivas posteriores, véase Walton, "Facetas de la corporalidad en la ética husserliana”, Eidos N.21 (2014), pp. 246-58. 
presencia primigenia) y la apercepción, destacando el carácter siempre parcial de la experiencia con los objetos. Al experimentar al otro como cuerpo, le transfiero por empatía las sensaciones vividas propias, en una coordinación de lo físico y lo psíquico.

En vistas del horizonte de cientificidad de sus análisis, destaca que solo en la experiencia objetiva del cuerpo del otro, como enlace y empatía mutua, puede manifestarse la realidad objetiva corporal y de la naturaleza, a través de la objetividad intersubjetiva de la cosa experimentada. De esta manera, se propone mostrar cómo, bajo la experiencia subjetiva del cuerpo y del mundo, subyace un estrato intersubjetivamente compartido (objetividades intersubjetivas), que permite el paso del sentido relativo al objetivo, tal y como lo despliega en este pasaje:

(...) La relatividad de las cosas de experiencia en referencia a los hombres singulares es por tanto indudable, y también es indudable que con la posición, que se ejecuta con la empatía, de un mundo intersubjetivo en la forma de la ciencia de la naturaleza, se abre la posibilidad de determinar "teóricamente" lo intersubjetivamente puesto, de tal manera que el contenido de determinación se vuelve independiente de los sujetos singulares o consiste exclusivamente en determinaciones que todo sujeto puede alcanzar a partir de sus daciones, tomándolas de las apariciones mediante el proceder metódico de la ciencia de la naturaleza, y con un sentido que es idénticamente el mismo para todo sujeto que investiga científico-naturalmente, y de tal manera que cada uno puede referir retrospectivamente las determinaciones a sus apariciones en su dependencia de su sujeto (Ideen II, pp. 170s. [212s])

Vemos aquí cómo esta doblez constitutiva permite comprender el pasaje de la esfera propia a la intersubjetiva. La experiencia del cuerpo propio y del cuerpo ajeno se torna aquí fundamental. En sus $M C$ podemos encontrar mayor precisión de los fenómenos de empatía y analogía. Entre tales especificaciones, destacamos a continuación un conjunto de supuestos que dan cuenta de algunas tensiones en este enfoque.

\section{1.d. El carácter especular y armónico en la experiencia corporal del otro}

En sus elaboraciones de 1931 encontramos amplios desarrollos y refuerzos de los análisis de los fenómenos intersubjetivos, en una vertiente gnoseológica. El punto de análisis se centra en una epojé egológica ${ }^{15}$, suspendiendo el marco intersubjetivo para reencontrarlo desde la esfera del ego puro $^{16}$. Del mismo modo, Husserl pondrá de manifiesto el paso hacia una

${ }^{15}$ Aquí seguimos la distinción de siete epojés en Husserl de Embree, "Seven Epoches", in Phenomenology and Practice N.5 (2011), pp. 119-126.

${ }^{16}$ En los Pariser Vorträge de 1929, base para sus Meditationen, Husserl hace mención de un autoconocimiento universal que pasa de su manifestación monádica (zunächst ei- 
conciencia trascendental intermonádica o comunidad trascendental, para dar cuenta de las manifestaciones culturales, espirituales y la naturaleza objetiva.

La corporalidad propia sigue un camino inverso al del conocimiento de la corporalidad ajena. En el primer caso, se pasa del cuerpo vivido a la manifestación del cuerpo físico; en el caso de la experiencia de los otros, se tiene en principio la corporalidad física y un conocimiento parcial y relativo de su cuerpo vivido y propio, cuyas vías vimos que son la empatía y la analogía. Husserl insiste en que tales vías no remiten a una inferencia del pensamiento, sino de una modalidad de experiencia con los objetos en el mundo, un fenómeno universal que se manifiesta dentro de la esfera primordial del ego $^{17}$.

Específicamente, en la percepción del otro, por medio de una apresentacion $^{18}$, se realiza una trasposición que liga aquel cuerpo al mío, por sus semejanzas. En este marco encontramos la modalidad predominante en la experiencia con las cosas naturales, la corporeidad del otro y de sí mismo: conocemos lo nuevo por esta vía de referencia a lo antiguo.

Del mismo modo la apercepción analógica permite percibir el cuerpo físico extraño como cuerpo orgánico. Esta instauración originaria es siempre presente: el ego y alter ego son dados en un originario emparejamiento (Paarung), presentándose como par en esta actualidad viviente, con una transferencia de sentido en los elementos apareados, lo cual remite a una asociación en una síntesis pasiva e intercambio mutuo de sentidos ${ }^{19}$.

Sin embargo, la unidad psicofísica del ego de aquel cuerpo percibido no puede llegar efectivamente a la presencia. Si lo percibiese directamente, su mundo pasaría a ser mi mundo y la analogía se convertiría en identidad constituida en mi ego (MC, pp. 138-9 [174]).Por ello, esta apresentación

ner monadischen) a una intermonádica (dann einer intermonadischen), en Husserl $P V$, pp. 39 s. [51s.].

${ }^{17}$ Husserl MC, pp. 141s. [178s.].

${ }^{18}$ Término que designa el estar co-presente un ser-también-ahí en el sustrato del mundo primordial.

${ }^{19}$ La síntesis pasiva designa aquella síntesis de unidades fenomenológicas que la conciencia encuentra ya hecha, es decir, que no procede de su actividad. Este concepto problemático en la teoría de Husserl, es un punto de partida fundamental para la recepción fenomenológica en los estudios actuales de neurociencias, así como también la apropiación por parte de las ciencias cognitivas. Véase Adrián, "La actualidad de la fenomenología husserliana: superación de viejos tópicos y apertura de nuevos campos de exploración”, Eidos N.18 (2013), pp. 30-4 y Rodríguez Vergara, "La conciencia de lo corporal: una visión fenomenológicacognitiva", Ideas y valores N.142 (2010), pp. 34-45, respectivamente. 
tiene un estilo de verificación distinto del caso de la percepción: el cuerpo ajeno se anuncia como orgánico solo en sus movimientos y comportamientos.

De esta manera, distinguimos en este enfoque un nexo especular: el otro se manifiesta como reflejo (Spiegelung) del ego, en la modalidad de analogía (Analogon), dándose así cierta duplicidad con lo ajeno. Esto se articula con algunas consecuencias del apareamiento y la empatía: comprendo su cólera o alegría, por ejemplo, a partir de mi propio comportamiento en similares circunstancias. A partir de la dinámica entre diferencia y similitud, toda comprensión lograda del otro abre nuevas posibilidades para su comprensión y la propia, de modo tal que el conocimiento de sí y del otro, siempre parcial, esta ajustado al devenir de la experiencia ${ }^{20}$.

Así, la comprensión de fondo se articula aquí en un enfoque gnoseológico de la tematización del otro ${ }^{21}$. No obstante, también despliega la cuestión relacional y comunitaria, con el objeto de evidenciar las remisiones a escalas mayores de tales enfoques y de la constitución intermonádica de la cultura y los valores ${ }^{22}$.

Sin embargo, estos análisis, al estar articulados en un modelo especular, permiten inferir tensiones en las dinámicas relacionales intersubjetivas, en el mismo marco donde Sartre y Lacan desarrollaron su comprensión conflictiva originaria de las relaciones con el otro. Frente a ello, Husserl apela al supuesto de una armonía entre las mónadas, a partir de la constitución co-correspondiente de la intencionalidad, en la copresencia intersubjetiva en el mundo de la experiencia ${ }^{23}$.

\section{1.e. Perspectivas y supuestos}

De esta manera, el punto de partida en estos análisis de la corporalidad propia y ajena confluye en tensiones al momento de dar cuenta de las relaciones intersubjetivas e intermonádicas. La perspectiva gnoseológica y ética de las elaboraciones que aquí analizamos parte de puntos de intersección y de modalidades universales de transferencia con base evidentemente especular, lo cual supone una concordancia mutua y normativa, junto a la comprensión del comportamiento del otro, que son difíciles de sustentar desde

\footnotetext{
${ }^{20}$ En torno a las tensiones que implican esta separación original yo-otros en su accesibilidad parcial, véanse los análisis en Waldenfels, "Fenomenología de la experiencia en Edmund Husserl”, Areté Vol.39, N.2 (2017), pp. 419ss.

${ }^{21}$ Para un desarrollo de esta interpretación, véase Presas, "La experiencia del otro", Escritos de Filosofía, N.12 (1983), p. 50.

${ }^{22}$ Véase Husserl MC, pp. 156s. ; 182 [198s. 232]. Cf. Husserl Ideen II, pp. 236-47 [283-95].

${ }^{23} \mathrm{Al}$ respecto, véase Husserl CM, p. 138 [173].
} 
la perspectiva fenomenológica que parte del ego puro $^{24}$. La consideración de un marco relacional armónico se nos presenta más bien como un supuesto relativo al horizonte de cientificidad hacia el que se orienta su análisis ${ }^{25}$.

Como veremos en el próximo apartado, el cambio de enfoque que propone y desarrolla Heidegger en sus elaboraciones en torno a la corporeidad, toma distancia de estas tensiones y limitaciones metafísicas, a partir de confluencias fundamentales con las elaboraciones de su maestro ${ }^{26}$.

\section{La corporeidad en Heidegger}

Los abordajes de la corporeidad (Leiblichkeit) en la obra de Heidegger se despliegan en su sentido ontológico. Un punto de partida posible podemos circunscribirlo en los tratamientos mínimos de la analítica en Sein un Zeit $(S u Z)$, donde destaca al Dasein en el mundo circundante ${ }^{27}$. De este modo, a diferencia del enfoque de Husserl, Heidegger parte del ocuparse con los entes desde la comprensión ya abierta en el mundo, tal y como se manifiesta en la estructura del ser-en-el-mundo (in-der-Welt-sein) ${ }^{28}$.

Respecto a este breve tratamiento, las distinciones de Sartre en su obra de 1943 son un posible antecedente para una tradición crítica respectiva, la cual parece descuidar la comprensión constelada implicada en la significación del término Dasein en la analítica ${ }^{29}$ y los requerimientos del proyecto heideggeriano de una ontología fundamental ${ }^{30}$. No obstante, podemos encontrar tratamientos más significativos en las clases de los Zollikoner

${ }^{24} \mathrm{El}$ supuesto de normatividad concordante en esta dimensión especular de la experiencia del otro, es analizado en Baldontín, "El problema de la constitución de la subjetividad en el pensamiento de Husserl", Alpha N.41 (2015), pp. 264s.

${ }^{25}$ Aquí seguimos los análisis de tensiones derivadas de esta comprensión de la alteridad de Rossi y Muñiz "Hermenéuticas de la intersubjetividad en Hegel y Husserl”, Revista Eletrônica Estudos Hegelianos Ano 9, N.16 (2012), p. 83 y Cataldo Sanguinetti, "Muerte y alteridad existencial en Martin Heidegger”, Eikasia. Revista de filosofía, 80 (2018), p. 202.

${ }^{26}$ Véase Dastur, Heidegger et la pensée à venir, J. Vrin, Paris, 2011, pp. 11ss.

${ }^{27}$ Para la consideración de menciones de la corporeidad en trabajos anteriores a SuZ, véase los análisis de Verano Gamboa, "El ser corporal: Dasein y lenguaje en el pensamiento de Martin Heidegger”, Convivium N.29/30 (2016-7), pp. 187-90.

${ }^{28}$ Véase un análisis de este cambio de enfoque en Inverso, "Heidegger frente a Husserl en la Introducción a la investigación fenomenológica”, Estudios de Filosofía, N.56 (2017), pp. 63-5.

${ }^{29}$ Véase Bauer, "Verstehen als Existenzial menschlichen Daseins ", Existenzanalyse Vol.33, N.1 (2016), pp. 11ss.

${ }^{30}$ En este sentido, véase las lecturas críticas de Adrián, "Heidegger y el olvido del cuerpo”, Lectora N.17 (2011), pp. 182ss. 
Seminare, donde podemos distinguir consonancias con los tratamientos de Husserl, pero con un claro cambio de enfoque.

2.a. Elaboraciones en torno a Sein und Zeit: la irreductibilidad del ser-en-el-mundo

En lo que respecta a $S u Z$, la tematización del cuerpo se desarrolla en el marco de las consideraciones de la espacialidad (Räumlichkeit) en su sentido originario. En efecto, en los parágrafos 23 y 24, Heidegger analiza la espacialidad del ser-en-el-mundo del Dasein y las relaciones de orientación y distancia con los otros cuerpos. Allí, frente a la comprensión moderna, destaca que al ser-en-el-mundo le es constitutivo abrir el espacio “en" el mundo, lo cual no implica que se halle en el sujeto, ni el mundo inscripto en el espacio (SuZ, p. 111 [136]). En tanto dado en la apertura de esta estructura, las cuestiones relativas a la espacialidad y las relaciones con el cuerpo se hallan inscriptas en el entramado de significaciones que liga el contexto respeccional, aquél en el que se constituyen las relaciones del Dasein con el medio y los objetos.

En este marco, la mención específica de la corporalidad se encuentra en sus análisis de la direccionalidad (Ausrichtung) y la des-alejación (Ent-fernung) (en sus vínculos con la lejanía (Ferne)). La estructura que da cuenta de ambas es el ser-en-el-mundo, lo cual permite destacar que la significación en esta estructura relacional del Dasein no se articula con la perspectiva instrumental propia del dualismo moderno sujeto-objeto: aquí, el habitar en el espacio cancela la posibilidad de comprender el cuerpo por fuera de sus relaciones en el mundo circundante.

Más en detalle: la desalejación implica un anular o disminuir la lejanía respecto a un punto de referencia. La relación con los demás entes se da en este carácter des-alejador del Dasein y desde allí es propicio evaluar las distancias en el mundo circunspectivo. Del mismo modo, lo a la mano (das Zuhandene) emerge en la ocupación circunspecta del Dasein, ámbito en el que se dirime el sentido de lejanía o cercanía (Nähe) del ente. Ello le permite a Heidegger subrayar que los sentidos corporales son co-extensivos con esta estructura ontológica: por ejemplo, el ver y oír son primariamente sentidos para lo lejano y luego, para lo cercano.

Esta estructura proyectiva de la espacialidad originaria también determina la direccionalidad: el Dasein primariamente se encuentra allí (en las dinámicas de des-alejamiento de los entes), accediendo luego a su aqui (al presente situacional del trato circunspecto). De este modo, tales coordenadas 
se hallan co-determinadas por el ser-en-el-mundo, lo cual supone un cuerpo orientado en el espacio ya abierto en esta modalidad originaria.

No obstante, Heidegger subraya que estas estructuras no permiten considerar objetiva la medición de distancias ni legitiman la referencia del espacio a una subjetividad rectora. Más bien, esto permite descubrir el ser-en-sí del ente en medio del cual el Dasein en cuanto existente está dado siempre (SuZ, p. 106 [132]), es decir, el entramado relacional en el que se abre la manifestación de los entes. En tal sentido, destaca que el Dasein no puede hacer un uso instrumental de sus des-alejaciones, ni cruzar su lejanía respecto a los entes, pues la desalejación es constitutiva en este entramado co-estructural originario.

De esta manera, nuestro autor presenta elementos de una comprensión de la corporeidad como modo originario de ser en el mundo, a partir de lo cual reconoce la necesidad de un análisis específico, por fuera de los límites propuestos para esta obra ${ }^{31}$. En línea con Husserl, cancela la posibilidad de pensar un existente humano sin un cuerpo, tomando como punto de partida esta irreductibilidad de la vinculación en el mundo circundante en su entramado respeccional de significatividad.

Ahora bien, la comprensión ya abierta del ser-en-el-mundo se torna un existencial fundamental para analizar las continuidades temáticas de esta ontología del cuerpo. En efecto, aquél entramado co-implica el comprender (Verstehen) que determina las cosas que experimentamos como significativas. En otras palabras, en la remisión circunspecta del Dasein ya actúa una (pre)comprensión del ser de los entes, la cual orienta las proyecciones y el modo de encontrarse entre ellos en el mismo horizonte de significatividad ${ }^{32}$. Asimismo, la comprensión del ser de los entes aquí implicada es una vía para dar cuenta de la comprensión del ser (Seinsverständnis) del Dasein en su época.

${ }^{31}$ Específicamente, subraya: El acercamiento no toma como punto de referencia la "cosa yo" dotada de un cuerpo, sino el ocupado estar-en-el-mundo, es decir, lo que en este estaren-el-mundo comparece inmediatamente. Por eso, la espacialidad del Dasein tampoco puede determinarse indicando el lugar en el que está presente una cosa corpórea (...) De esta direccionalidad provienen las direcciones fijas hacia la derecha y la izquierda. Al igual que sus des-alejaciones, el Dasein lleva también constantemente consigo estas direcciones. La espacialización del Dasein en su "corporalidad", que implica una problemática propia que no ha de ser tratada aquí, se halla también caracterizada por estas direcciones. (Heidegger SuZ, pp. 107s. [133s.]).

${ }^{32}$ Sus elaboraciones de la comprensión se consolidan en relación con la proyección del Dasein, en Heidegger SuZ, pp. 86ss. [112ss.]; 142-54 [166-77]. 
De esta manera, comprensión y significatividad son correlativas al seren-el-mundo y dan cuenta de las modalidades de aprehensión de las cosas y de proyección en las relaciones factuales. Ello nos permite distinguir ciertas continuidades con el perspectivismo que hemos visto en los análisis de Husserl: los desplazamientos comprensivos y de significatividad son correlativos a desplazamientos en la manifestación remisional y el ocuparse en el trato circunspecto ${ }^{33}$. Precisamente por ello, una indagación en los modos de presentación de los entes permite dar cuenta de la comprensión de ser ya abierta en una época. Distinguimos aquí el camino hacia una ontología fundamental en la analítica de Heidegger y una de las razones centrales del breve tratamiento que recibe aquí la corporeidad.

Estas cuestiones indican los caminos de Heidegger en sus trabajos de madurez, en especial, en lo que respecta a sus consideraciones en torno la historia del ser, la época del mundo de la técnica y las posibilidades respectivas de transición. Tal es el caso, por ejemplo, de la mención en la analítica del ser-a-la-mano (Zuhandenheit) de lo útil, como otra de las estructuras del ser-en-el-mundo, donde resuena la relación entre cuerpo y técnica de sus trabajos posteriores.

\section{La corporeidad en los Zollikoner Seminare}

Ahora bien, a partir de la escueta tematización en SuZ, Heidegger se abstuvo de desarrollar una fenomenología del cuerpo sin antes determinar con claridad la comprensión adecuada al Dasein que ella requiere, es decir, en su respectiva diferencia ontológica y sus estructuras específicas ${ }^{34}$.

Siguiendo aquí a Husserl, lo que distingue como la corporalidad viva (Leiblichkeit) requiere una aprehensión ontológica que advierte como ausente en la tradición metafísica, centrada principalmente en la corporalidad cósica (Körperlichkeit). Así, su labor de análisis en torno al modo de ser del Dasein tiene como correlato una reelaboración de la tematización existencial del cuerpo propio.

${ }^{33}$ Sobre las discusiones de Heidegger con las obras de Husserl desde 1920, véase Volpi, "Ser y tiempo: semejanzas con la Ética Nicomaquea", Signos filosóficos, Vol.VIII, N.16 (2006), pp. 131ss.

${ }^{34}$ Esta es su posición respecto de las críticas de Sartre: insiste en que es necesario un desarrollo del existenciario del ser-en-el-mundo y, solo desde allí, una descripción suficiente del fenómeno de cuerpo. Asimismo, distingue una diferencia fundamental con el enfoque de Sartre, quien ve al ser como algo establecido en un proyecto subjetivo del ser humano (Heidegger ZS, pp. 202ss. [240ss.]; 277 [321]). 
Específicamente, en los Zollikoner Seminare (ZS) de 1959-1969, Heidegger busca dar cuenta de un sentido originario de la corporeidad que no se agote en el cuerpo mensurable y objetivable (Körper) de algo meramente allí presente (vorhanden). Tal determinación e indistinción de la ciencia natural lo hizo coincidir con el cuerpo que experimentamos como propio ${ }^{35}$.

Estos seminarios se originan en un intercambio epistolar con Medard Boss desde 1947, donde se propusieron especificar y comprender las cuestiones inherentes al análisis del Dasein. En ese marco, Boss lo invito a unas conversaciones con un conjunto de colegas y estudiantes de psiquiatría y psicología en su casa en Zollikon, desde 1959. Lo que Heidegger promovió en estos encuentros es la experiencia de un pensar, ver y decir otro, respecto a las cuestiones consideradas.

En efecto, en los primeros encuentros subraya que el existente humano ya no puede reducirse a mero yo o sujeto, pues ello oculta se apertura al mundo y sus correlaciones. Podemos sostener que el propósito central de tales encuentros es demostrar que la noción médica de cuerpo aún se maneja en los límites de las coordenadas cartesianas ${ }^{36}$ : el Dasein, en tanto ente sostenido en/por el Ereignis, es decir, comprendido de un modo irreductible desde la relación con aquello que se desoculta desde la apertura del mundo, no puede ser reducido en su aspecto material y presente, tal y como se ha caracterizado en el abordaje tradicional.

En este marco, los análisis que realiza en torno a la corporalidad, el tiempo y el espacio, tienen un desarrollo marcadamente descriptivo, pues buscan despejar las condiciones para un análisis más pertinente que ponga de manifiesto el estatuto ontológico respectivo. En tal sentido, Heidegger rechaza la posibilidad de una fenomenología del cuerpo, debido al carácter entramado de la constitución del cuerpo ${ }^{37}$, lo cual puede interpretarse como una vía indirecta para dar cuenta del método genuino y pertinente de abordaje ${ }^{38}$. A continuación, analizaremos su posición respecto a la perspectiva

${ }^{35}$ Salvo los casos donde se especifique $\operatorname{Körper}(\mathrm{K})$, a continuación nos referiremos a cuerpo en tanto Leib. En este marco, aquí utilizaremos los términos corporalidad y corporeidad, en referencia a manifestaciones perceptibles del cuerpo y a las estructuras ontológicas del mismo, respectivamente.

${ }^{36}$ Interpretación que seguimos de Adrián "Heidegger y el olvido del cuerpo", Lectora N.17 (2011), pp. 184s.

${ }^{37}$ Véase Heidegger ZS, p. 231 [272].

${ }^{38}$ Véase Johnson, "La exclusión del cuerpo en Sein und Zeit y la negación de una fenomenología del cuerpo en el pensamiento de Heidegger", Pensamiento Vol.72, N.270 (2016), pp. 132ss. 
científica tradicional, con el objeto de dar cuenta de la comprensión del ser-en-el-mundo que le subyace. En contraposición, desarrollaremos luego las descripciones de fenómenos corporales que nuestro autor propone para dar cuenta de tal estatuto: las cuestiones relativas a la relación espacialidadcuerpo y algunas figuraciones de la corporeidad en la localización corporal de los sentidos. Finalmente, desarrollaremos sus consideraciones de los vínculos con el cuerpo ajeno, en el marco de su posición crítica con el enfoque de Husserl. Como veremos, el carácter originario de la comprensión y los nexos de significatividad atraviesan y modalizan toda manifestación corporal, lo cual le confiere un estatuto primario al momento de tematizar y analizar la corporeidad.

\section{3.a. Limitaciones en la comprensión científica}

Los ejercicios del pensar que propone Heidegger en dichos encuentros consisten en percibir desde lo que se muestra, destacando un ámbito previo a la mirada escrutadora de la ciencia dirigida a los fenómenos inmediatamente perceptibles. Los estratos de la experiencia fáctica muestran su carácter respectivo, es decir, de ligazón con diversas estructuras y ámbitos, más allá de la mera manifestación presente en lo aparentemente aislado o autónomo.

En tal sentido distingue que la representación científica aplica criterios de legitimidad que derivan en la calculabilidad de la naturaleza, considerando solo aquello susceptible de cuantificación en el ámbito de lo que es. En la misma línea, la ciencia natural constata al ser humano como algo que esta simplemente ahí en la naturaleza, solo considerado como un ente natural.

La mensurabilidad, fundamentada en el carácter extenso y presente del ente, es correlativa a un comportamiento del ser humano. Pertenece a la cosa representada en cuanto objeto ${ }^{39}$, comprensión de partida para estas relaciones. En este sentido, la objetualidad es una determinada modificación de la presencia de las cosas: la presencia es entendida como representacionalidad, no ya como lo dado a partir de sí, sino tal y como se nos contrapone en tanto sujetos. Por ello, la mensurabilidad deriva en calculabilidad,

${ }^{39}$ El término objeto tiene en los desarrollos de Husserl y Heidegger una importante distinción. Como en el empeño teórico metafísico se incluye la pregunta por la condición de posibilidad del conocimiento en general, cabe diferenciar lo cognoscible según dos modalidades: a) Gegenstand, traducible como "lo enfrentado", en referencia a aquello con que nos encontramos en el mundo y b) Objekt, en referencia al objeto de conocimiento de las ciencias, es decir, a los primeros en tanto configurados según las exigencias de las ciencias. En este trabajo, haremos especial referencia al objeto, cuando nos remitamos a su procesamiento dentro de la comprensión y experiencia científica. 
una observación de la naturaleza que requiere saber con qué podemos contar de sus procesos. Esto implica a su vez una precalculabilidad, es decir, un proceder de dominio y posesión sobre los procesos naturales, lo cual señala otra co-implicancia: el supuesto de determinaciones específicas de lo ente y de una subjetividad rectora ya implícita en esta modalidad de comprensión.

Heidegger destaca que tal comprensión es moderna y específicamente cartesiana: lo ente solo es tal si es representado o representable, modificando la experiencia de la presencia del ente hacia la objetualidad.

Ahora bien, tal fijación anticipable de la naturaleza conlleva algunas consecuencias previsibles: aquello que no muestre el carácter de objeto posible de determinación matemática es suprimido como incierto, como no siendo verdadero. Más aún, lo que es existente solo es considerado a partir del tipo de verdad fijada por el sentido de certeza, relativo a la subjetividad representativa cartesiana. En este marco, sostiene:

"La ciencia así fijada, esto es, este método, es el ataque más monstruoso del ser humano a la naturaleza (...)habla una dictadura del espíritu que rebaja el espíritu mismo a un operador de la calculabilidad y así hace valer su pensar solo como un manipular con conceptos operativos y representaciones de modelos y modelos de representación ; no solo hace valer, sino que se atreve a hacer pasar, en una ceguera monstruosa, a la conciencia reinante en esta ciencia, incluso como la conciencia crítica” (ZS, p. 139 [174]).

Es precisamente en el marco de esta experiencia moderna desde donde nuestro autor cuestiona la medida en que el fenómeno del cuerpo resiste la mensurabilidad, a partir de la cual indaga en torno a métodos adecuados para la determinación e interpretación del cuerpo en sus manifestaciones.

En línea con estas distinciones, Heidegger se posiciona críticamente respecto del imperativo altruista médico y su comprensión subyacente: en lo que respecta al existente humano siempre se trata del existir y no del funcionar de algo. Aun así, respecto al dominio de lo terapéutico, subraya que el ser humano necesita ayuda pues siempre está en peligro de perderse, de no poder consigo mismo, aspecto en estrecha vinculación con la libertad humana: cada enfermedad es una pérdida de libertad, una limitación de la posibilidad de vivir.

En suma, las distinciones co-estructurales y simultáneas que se hallan imbricadas en los fenómenos del cuerpo permiten reelaborar el pensar psicopatológico y psicológico, desde un paradigma experiencial que se desplaza del pensar representativo y de aquella experiencia objetual científico-natural, es decir, de su modalidad específica de ser-en-el-mundo con su comprensión respectiva. 


\section{3.b. Figuraciones de la corporeidad}

Diversos son los puntos de indagación respecto al problema del cuerpo en estas conversaciones en Zollikon, a saber: la pregunta de la psicosomática, las relaciones cuerpo-inconsciente; las implicancias psíquicas; los fenómenos no sustancialistas del cuerpo (como las lágrimas, el ruborizarse por vergüenza), las consideraciones espaciales y de los sentidos, etc. Aspectos que, tradicionalmente delimitados como mensurables, revelan dimensiones co-determinantes como las relaciones con los otros y con el entorno, los éxtasis temporales, la memoria, entre otras. Estas referencias remiten al carácter extático del Dasein, por lo cual no son representables. Tan pronto como nos las representamos, nos colocamos fuera de la referencia extática.

Por este motivo, estas indagaciones se despliegan en el marco de la distinción entre el análisis del Dasein (Daseinsanalyse) y la analítica del Dasein (Daseinsanalytik). Esta última, en tanto ontológica, articula la unidad de un compuesto estructural dinámico, que se manifiesta anterior o simultáneo al análisis óntico del Dasein. Desde aquí, Heidegger aborda las consideraciones del cuerpo en torno a dos estructuras correlativas: el ser-en-el-mundo y el ser-con. En ambos casos, sobre el trasfondo de una apertura comprensiva del ser. Con el objeto de dar cuenta con mayor precisión de su carácter correlativo, abordemos algunas de sus descripciones.

\section{3.b.I. Relaciones en la espacialidad}

Heidegger retoma sus consideraciones de la espacialidad en SuZ, analizándola en su carácter respectivo y relacional. Por un lado, aborda la consideración de la extensión y la experiencia del límite del cuerpo. Distingue que, si bien el cuerpo $(\mathrm{K})$ termina en la piel, estamos siempre en relación con algo más allá del cuerpo. Este límite se manifiesta con mayor extensión que en el caso del cuerpo físico. Hay entre ambos una diferencia cualitativa: aquella extensión remite al horizonte de nuestro ser-en-el-mundo y no a lo meramente mensurable del espacio. El límite del cuerpo $(\mathrm{K})$ generalmente no cambia, tal y como podría figurar en fenómenos tales como engordar o adelgazar.

En este marco, retoma la consideración de la lejanía y el carácter desalejador del Dasein en su vínculo corporal con los entes ${ }^{40}$. El carácter situacional del aquí del cuerpo va más allá de la materialidad implicada en el espacio: implica estar ocupado, sumido en una cosa, de modo tal que el cuerpo habita, hace estancia (Aufenthalt) en el espacio, es decir, participa en un entramado remisional que pone de manifiesto y abre la estructura del

\footnotetext{
${ }^{40}$ Véase Ibíd, pp. 109ss. [142ss.].
} 
ser-en-el-mundo. En este sentido, distingue el estar en el espacio del cuerpo y la orientación del Dasein, de la mera espacialidad cósica.

Por otro lado, ejemplifica estas cuestiones a partir del fenómeno de la presencialidad o el hacer-presente (Vergegenwärtigung) (aquél por medio del cual se hace presente algo en la experiencia), a través del ejercicio de hacer (se) presente la estación central del tren de Zúrich ${ }^{41}$. Allí se revela un fenómeno distinto a la mera representación (Vorstellung) o imagen de la cosa (como en el caso del recuerdo), que pone de manifiesto un co-estar con ella, por medio de lo cual ésta se muestra desde diversas perspectivas. En tal mostrarse, la cosa se halla presente en sus posibilidades de hacerse presente, dando por sí misma las indicaciones y pruebas necesarias de aquello que se manifiesta. Asimismo, al presentificarla nos encontramos en medio de (Sein bei) la cosa, ya no como aquel fenómeno que se da en el simple imaginarse (Sich-einbilden), sino en la modalidad de ser-ahí con el ente en la experiencia. Las especificaciones de este fenómeno permiten dar cuenta, por un lado, de la co-implicación de la presencia corporal del ente y del Dasein (en su corporeidad) y, por otro, de una modalidad del pensar no representativo que se caracteriza por su apertura (Offenständigkeit) ${ }^{42}$.

Por ello, el ser corporal en el mundo implica que el hombre se halla abierto a las modalidades del darse de las cosas. El ser-aquí (Hiersein) ejecuta la presencialidad en distintas modalidades de estar en medio de, lo cual tiene el carácter del percibir corporal cosas que están abi en persona (ZS, 94 [126]). En línea con aquel carácter desalejador elaborado en SuZ, aquí nuestro autor subraya que el ser-aquí en medio de las cosas es también siempre un ser-allá (Dort-sein) con las cosas distantes, que implica un serabierto para lo presente con que se está de un modo diferencial respecto a la mera reunión corporal física de dos cosas en el espacio. Esta apertura distingue la corporeidad y co-existencia del Dasein en medio de los entes.

\section{3.b.II. Los sentidos y los movimientos de la mano}

En la misma línea, nuestro autor aborda algunas consideraciones respecto a los sentidos: el ver y oír dirigidos al mundo; al agarre o el tocar; el quedar absorto en una cosa, donde el cuerpo se manifiesta en ausencia

\footnotetext{
${ }^{41}$ Véase Ibíd, pp. 86-97 [118-129].

${ }^{42}$ Heidegger especifica que en el hacer-presente podemos estar fácticamente en otro lugar respecto a la cosa (por ejemplo, estar sentados en el seminario mientras se presentifica la estación de Zúrich), pero la presentificación establece el modo en que se está en dicho lugar (es decir, en el modo de hacer-presente la estación) implicando, por ejemplo, cierta desatención de las cosas allí presentes, en Ibíd, p. 93 [125s.]).
} 
o como fenómeno de privación, etc. También analiza los fenómenos de las lágrimas, el ruborizarse, el dolor, es decir, un conjunto de manifestaciones corporales donde se ponen de manifiesto sus respectivos entramados interrelacionales y de significación.

$\mathrm{Al}$ igual que Husserl, encuentra en la experiencia de la mano una mayor posibilidad de aprehensión que en el caso del sentido auditivo o visual, pues permite establecer un contacto directo con lo agarrado (el ojo, en cambio, no está en contacto directo con lo visto $)^{43}$. Aquí, el sentido de proximidad (Nab-Sinn) relativo al tacto, se opone al sentido de la lejanía (Fern-Sinn) en el ver, de modo correlativo a la aprehensión del cuerpo: en la experiencia, éste es lo más lejano en el espacio.

En este marco, se posiciona críticamente a la concepción tradicional del gesto (Gebärde) en el sentido de expresión (Ausdruck), que se halla implicada en la representación del cuerpo de la tradicional significación griega o latina: un movimiento de la mano, como saludar, pertenece al ser de la mano y no simplemente indica una expresión de interioridad. En tal sentido, destaca que el movimiento mismo de la mano ya es el significado del saludar y no la remisión a un estado mental o afectivo ${ }^{44}$.

Con la explicitación de esta manifestación, Heidegger subraya el carácter constitutivo de cuerpo y espacio, lo cual le permite oponerse al dualismo internalismo-externalismo y la relación sujeto-objeto de los análisis tradicionales de las manifestaciones físicas: para nuestro autor, el sentido del ser corporal es correlativo a las relaciones en el mundo circundante. Así, el gesto, remite al comportarse (Betragen) del hombre como ser corporal en el mundo, en su relación irreductible con los otros entes.

Aquella imbricación también figura en la expresión somos todo oídos en una conversación: corporalmente participamos en el diálogo, co-ejecutando el diálogo por el oír-hablar con el que participo, por ejemplo, al estar sentado en la silla para poder ser todo oídos ${ }^{45}$. Subraya así el sentido extático de la corporalidad. Asimismo, en este análisis del gesto también se evidencian las relaciones de significación en la comprensión, donde cuerpo y espacio se entretejen: el significado de un gesto se articula en las relaciones con los entes, es decir, remiten a modalidades del ser-en-el-mundo.

${ }^{43}$ Ibíd, pp. 107ss. [141ss.].

${ }^{44}$ Véase Ibíd, pp. 116-9 [150-3].

${ }^{45}$ Ibíd, pp. 125s. [160].Esta distinción permite desplegar una lectura sobre el carácter corporal del lenguaje, especialmente en sus desarrollos en Unterwegs zur Sprache, tal y como podemos encontrar en Verano Gamboa, "El ser corporal: Dasein y lenguaje en el pensamiento de Martin Heidegger", Convivium N.29/30 (2016-7), pp. 197-201. 


\section{3.c. La inter-corporeidad desde el ser-con}

En el marco de esta modalización que imprime la comprensión, nuestro autor destaca que el corporar (Leiben) se determina a partir del modo del ser del Dasein ${ }^{46}$. El corporar, como modalidad de manifestación del cuerpo en tanto es cuerpo, pertenece siempre al ser-en-el-mundo pues lo co-determina el estar-abierto, el tener mundo. El límite del corporar es el horizonte del ser en el cual permanecemos. Por ello, este límite cambia mediante la modificación del alcance de mí habitar y estar en el espacio. Todo el comportamiento del ser humano en cuanto ser-en-el-mundo está determinado por el corporar del cuerpo, incluso todo movimiento de mi cuerpo en cuanto gesto y el comportarse, los cuales están ya siempre en una región abierta por la cosa con la que estoy relacionado. Dos son las co-estructuras que nuestro autor distingue en tal modalización: el ser-con (Mit-sein) y el comprender. Desde allí cancela la posibilidad de lecturas subjetivistas de esta consideración corporal del Dasein ${ }^{47}$.

En este marco, destacamos el carácter ejemplificador del análisis de un caso de psicopatología en el intercambio epistolar con Boss. Allí, retoman un caso de tipo freudiano: una mujer que olvida la bolsa al irse de la casa de un conocido, lo cual tradicionalmente se ha interpretado como un deseo inconsciente de poder regresar ahí. Frente a ello, Heidegger rechaza la intención inconsciente y sostiene: "su irse es tal que en el irse ella todavía y con mayor razón todavía y siempre más está-ahí. Porque ella, en el irse, está todavía tanto con el hombre, que la bolsa no está en absoluto ahí” (ZS, pp. 213s. [252]). Tenemos aquí una extensión y un corporar que ponen de manifiesto una estructura relacional irreductible y no mensurable.

Precisamente desde esta comprensión busca apartarse de las elaboraciones de Husserl respecto a la inter-corporalidad y sus modalidades de acceso a la dimensión del cuerpo ajeno.

Específicamente, en la sesión de julio de 1965 analiza la cuestión de la relación inter-corporal, tomando distancia de las elaboraciones respectivas de Husserl en MC, aunque destacándola como su obra fundamental (ZS, pp. 142-6 [177-82]). Sostiene que hablar de relación interhumana o cohumana orienta el pensar hacia una representación dualista de dos sujetos que están-ahí. Incluso, el concepto mismo de relación obstaculiza aprehender el auténtico vínculo que se manifiesta entre el Dasein y los otros. Con

${ }^{46}$ Véase Heidegger ZS, p. 113 [147].

${ }^{47}$ Véase Ramírez Cobián, "El cuerpo por sí mismo. De la fenomenología del cuerpo a la ontología del ser corporal”, Open insight Vol.8, N.14 (2017), pp. 223s. 
ello, toma distancia del constructo implícito en la teoría husserliana de la empatía, en la cual un yo dado primariamente para sí, empatiza luego en el otro, descubriéndolo como alter $\mathrm{ego}^{48}$.

Frente a ello, Heidegger destaca la estructura del ser-con, entendida como existir con otros en la forma de ser-en-el-mundo, desde un mutuo referirse a las cosas en la experiencia ${ }^{49}$. Es decir, el ser-unos-con-otros-en-el-mundo es la estructura interrelacional del Dasein, que implica el encontrarse con otros en un mismo ser-ahi ${ }^{50}$. Ello no remite a una relación externa entre sujetos, de uno a otro. El mero encuentro entre cuerpos físicos es privación de esta estructura ontológica, donde los existentes no tienen nada que ver uno con otro. En este sentido, subraya que la proyección supuesta de la empatía ya presupone el ser-con en las relaciones mutuas y la comprensión del otro como ser humano. No obstante, desde esta crítica a la perspectiva inicial de Husserl, nuestro autor destaca el posterior abandono de su perspectiva cartesiana, especialmente por medio de la referencia originaria al mundo de la vida (Lebenswelt) en sus trabajos de madurez ${ }^{51}$.

Finalmente, Heidegger insiste en el carácter originario y primario de la comprensión respecto a estas estructuras interrelacionadas. En tal sentido, el ser-en-el-mundo no se agota en el corporar que se manifiesta allí donde la sensibilidad se halla presente: subraya que en el horizonte de la comprensión del ser no sucede ningún corporar (ZS, p. 244 [286]). En este horizonte se evidencia cómo el ser está determinado por y en la comprensión.

De este modo, la distinción primaria y originaria de la comprensión permite destacar las prioridades específicas en lo que respecta a estos análisis de la corporeidad: entre otras implicancias, distinguimos aquí el carácter

${ }^{48}$ No obstante, aquí también distinguimos continuidades con las elaboraciones de su maestro a partir de las lecturas de Zahavi sobre la intersubjetividad a priori en Husserl, en las cuales pone énfasis en la cuestión de la constitución por sobre la empatía, en Zahavi, Husserl and Transcendental Intersubjectivity, a Response to the Linguistic-Pragmatic Critique, Behnke, E. (trad.), in Athens: Ohio University Press, 2001, pp. 154ss. Cf. Cabrera, "Intersubjetividad a priori y empatía", Ideas y valores Vol.62, N.152 (2013), pp. 81-92.

${ }^{49}$ Este carácter constitutivo de la alteridad en sus conexiones con elaboraciones de Husserl es analizado en Candiotto, "Heidegger's ontic relatedness: Pros ti and Mitsein", Aurora, Rev. de filosofía, Vol.28, N.43 (2016), pp. 319ss.

${ }^{50}$ Podemos encontrar lecturas de estas co-implicancias que permiten sustentar un empuje comunitario constitutivo en Haar, "Stimmung et pensée" en Heidegger et l'idée de la phénoménologie. Dordrecht: Kluwer Academic Publishers 1998, pp. 268ss.; Rossi, "El problema de la comunidad en Ser y tiempo", en Lythgoe-Rossi, Ser y tiempo. Singularización y comunidad, Buenos Aires, Biblos, 2016, pp. 140ss.

${ }^{51}$ Véanse los comentarios sobre las elaboraciones de Husserl en Heidegger ZS, p. 187 [224]; 207 [246].Cf. Husserl DL, pp. 60-5; 175-8; 200-5, etc. 
secundario o derivado de los fenómenos corporales del Dasein, en el marco de las dinámicas comprensivas originarias e históricas. No obstante, la falta de un desarrollo de la función vitalista del cuerpo bien puede interpretarse como un modo de manifestar ciertos compromisos con las consideraciones objetivistas del cuerpo ${ }^{52}$.

\section{Consideraciones finales}

Para terminar proponemos un conjunto de puntualizaciones en nuestro recorrido por estas consideraciones sobre el cuerpo, con el objeto de evidenciar enfoques, confluencias y continuidades.

En primer lugar, con el enfoque desarrollado en dos obras de Husserl, tomando como punto de partida la esfera primordial del ego y el cuerpo propio, describe manifestaciones co-constituyentes que remiten al otro, no solo en lo que respecta al despliegue y la constitución de lo cultural e institucional, sino también en su estatuto corporal, desde una perspectiva principalmente gnoseológica. Siendo la empatía y las apercepciones analógicas los medios para dar cuenta de tales correlaciones intersubjetivas, vimos algunas tensiones resultantes de un modelo de análisis que distinguimos como marcadamente especular. En ese marco, colegimos que el supuesto de una armonía monádica, basado en la universal constitución de la intencionalidad, puede entenderse como resultado de los imperativos de cientificidad que orientan sus investigaciones. Si bien recepciones contemporáneas de la fenomenología, como el caso de Zahavi, distinguen la intersubjetividad a priori como dimensión originaria que se revela con el despliegue de este método, entendemos que la orientación descriptiva tensiona las consideraciones respectivas. Entre ellas, destacamos el carácter parcial u opaco del conocimiento de la esfera (propia) del otro y su cuerpo, que solo cuenta con las perspectivas otorgadas por experiencias especulares desde las relaciones inter-corporales. En este sentido, frente a sus elaboraciones del Leib social y el supuesto de una co-constitución armónica, podemos inferir una dimensión relacional intersubjetiva de tipo antagónico, derivada de la base especular de la experiencia del otro. Precisamente por estas tensiones, los despliegues fenomenológicos de Husserl y sus resultados permiten distin-

${ }^{52}$ Aquí seguimos la lectura propuesta por Llorente, "Heidegger y el estatuto ontológico del cuerpo. Una confrontación con la fenomenología de la carnalidad de Michel Henry”, Ideas y Valores Vol.65, N.162 (2016), pp. 265-75. 
guir en ello condiciones para una transición en las consideraciones filosóficas de la corporalidad ${ }^{53}$.

En segundo lugar, abordamos dos momentos del tratamiento de la corporeidad en los trabajos de Heidegger, los cuales entendemos como pasos fundamentales para dicha transición. Aquí, las indagaciones se despliegan para dar cuenta de ámbitos estructurales originarios de la constitución ontológica del Dasein: el carácter entramado de lo corporal, el ser-en-el-mundo y el ser-con. En el caso de $S u Z$, los análisis de la espacialidad permiten dar cuenta por un lado, del entramado irreductible de Dasein-cuerpo-mundo y, por otro, del rechazo a un abordaje subjetivista e instrumental de la relación entre los cuerpos. En tal sentido, destaca el carácter originario de la comprensión y los nexos de significatividad en los fenómenos considerados, lo cual torna imprescindible una indagación sobre la comprensión del ser que orienta y modaliza las relaciones consideradas. Por esta vía, años después desarrolla descripciones específicas de manifestaciones corporales.

En efecto, en los seminarios de $Z S$, vimos que nuestro autor despliega con más detalle las estructuras relativas a la corporeidad, en el marco de sus análisis de la tradición científica y su comprensión cósica del cuerpo, cuya modalidad de ser-en-el-mundo se destaca por la calculabilidad, instrumentalización, control y dominio en las relaciones con el entorno. En este marco, con su rechazo explícito a indagar primariamente por la vía de una fenomenología del cuerpo, pone de manifiesto el carácter interrelacionado del ser corporal del Dasein y la primacía originaria de la comprensión. Con ello deja entrever, por un lado, que una perspectiva originaria de la corporeidad debe primero dilucidar con precisión las cuestiones relativas a la comprensión del ser que modalizan el ser-en-el-mundo y, por otro, las limitaciones prácticas que tiene el ser-corporal del Dasein, en lo que respecta a las injerencias instrumentales en el ámbito de la comprensión. Desde aquí pueden recorrerse sus consideraciones sobre la praxis y la ética en sus trabajos de madurez.

En suma, los abordajes de la inter-corporalidad de Husserl ponen de manifiesto ciertas confluencias entre lo cultural, lo espiritual y lo ético que no logran escapar de los atolladeros y las tensiones propias de los dualismos metafísicos tradicionales. No obstante, los resultados de sus análisis ofrecen elementos que clarifican el lugar que ocupa la intersubjetividad en la

${ }^{53}$ Sobre el aspecto transicional de Husserl respecto a la perspectiva de Descartes, véase Staiti, "The Pedagogic Impulse of Husserl's Ways into Transcendental Phenomenology”, Graduate Faculty Philosophy Journal, N.33.1 (2012), pp. 40ss. 
constitución de la esfera primordial del ego, lo cual se vincula con sus posteriores análisis del mundo de la vida. Precisamente este es el punto de partida de las elaboraciones de Heidegger, donde el carácter entramado y correlativo de las estructuras del ser corporal del Dasein pone en evidencia la necesidad de una indagación en la comprensión y las remisiones de significación epocales. En ese marco, el carácter relacional Ser-Dasein y la comprensión allí implicada reducen el margen para la injerencia práctica en las dinámicas epocales del ser. En este punto, la corporeidad y la comprensión se entretejen por fuera de los imperativos instrumentales de la metafísica de la subjetividad: quizás podamos reconocer aquí el punto cero de una transición en los abordajes y tematizaciones en torno al cuerpo.

\section{Bibliografía}

Adrián, J., "Heidegger y el olvido del cuerpo", Lectora N.17 (2011), pp. 181-98.

Adrián, J., "La actualidad de la fenomenología husserliana: superación de viejos tópicos y apertura de nuevos campos de exploración”, Eidos N.18 (2013), pp. 12-45.

Baldontín, C., "El problema de la constitución de la subjetividad en el pensamiento de Husserl", Alpha N.41 (2015), pp. 251-68. https://doi. org/10.4067/S0718-22012015000200017

Bauer, E., "Verstehen als Existenzial menschlichen Daseins », Existenzanalyse Vol.33, N.1 (2016), pp. 4-14.

Cabrera, C., "Intersubjetividad a priori y empatía", Ideas y valores Vol.62, N.152 (2013), pp. 71-93.

Candiotto, L., "Heidegger's ontic relatedness: Pros ti and Mitsein", Aurora.Revista de filosofía, Vol.28, N.43 (2016), pp. 313-328. https://doi. org/10.7213/aurora.28.043.AO03

Cataldo Sanguinetti, G., "Muerte y alteridad existencial en Martin Heidegger", Eikasia, Revista de filosofía, N.80 (2018), pp. 199-217.

Dastur, F., Heidegger et la pensée à venir, J. Vrin, Paris, 2011.

Derrida, J., El tocar, Jean-Luc Nancy, Buenos Aires, Amorrortu, 2011.

Embree, L., "Seven Epoches", Phenomenology and Practice N.5 (2011), pp. 120-6.

https://doi.org/10.29173/pandpr19848

Haar, M., "Stimmung et pensée » en Heidegger et l'idée de la phénoménologie. Dordrecht: Kluwer Academic Publishers (Phaenomenologica Vol.108), 1988. 
Heidegger, M. [LFW], "Logik. Die Frage nach der Wahrheit (Winter semester 1925/26)", en Gesamtausgabe 21, ed. W. Biemel [Trad. Cast.: Lógica. La pregunta por la verdad, Alianza Editorial, Madrid, 2004, trad. J. A. Ciria], 1925-6.

Heidegger, M. [SuZ], "Sein und Zeit", en Gesamtausgabe 2 (Trad. cast: Ser y Tiempo, Santiago, Ed. Universitaria de Chile, 1997, Trad. J.E. Rivera; Ser y tiempo, Buenos Aires, Fondo de la Cultura Económica, 2000, trad. J. Gaos), 1927a.

Heidegger, M. [DGPh], "Die Grundprobleme der Phänomenologie", en Gesamtausgabe 24 [Trad. cast.: Los problemas fundamentales de la fenomenología, Madrid, Trotta, 2000], 1927b.

Heidegger, M. [US], "Unterwegs zur Sprache”, en Gesamtausgabe 12 [Trad. cast.: De camino al Habla, Barcelona, Serbal, 2002, trad. Ives Zimmermann], 1950-1959.

Heidegger, M. [ZS], “Zollikoner Seminare”, en Gesamtausgabe 89 [Trad. cast.: Seminarios de Zollikon, Barcelona, Herder, 2014, trad.: Ángel Xolocotzi Yáñez], 1959-69.

Husserl, E. $[P V]$, "Pariser Vorträge" en Cartesianische Meditationen und Pariser Vorträge, Ed. S. Strasser. La Haya, Netherlands: Martinus Nijhoff [Trad. cast.: Las conferencias de París, Instituto de Investigaciones Filosóficas, Cuaderno N.48, UNAM, 1988], 1950.

Husserl, E. [Ideen II], "Ideen zur einer reinen Phänomenologie und phänomenologischen Philosophie II: Phänomenologische Untersuchungen zur Konstitution", en Husserliana 4, Ed. Marly Biemel. La Haya, Netherlands: Martinus Nijhoff [Trad. cast.: Ideas relativas a una fenomenología pura y a una filosofía fenomenológica II: Investigaciones fenomenológicas sobre la constitución, México, Fondo de la Cultura Económica, 2003, Trad: Zirión, Antonio], 1952 [1913].

Husserl, E. [CM], "Cartesianische Meditationen und Pariser Vorträge", en Husserliana 1, Ed. S. Strasser. La Haya, Netherlands: Martinus Nijhoff [Trad. cast.: Meditaciones Cartesianas, Madrid, Ediciones Paulinas, 1979, Trad.: Mario Presas], 1973 [1931].

Husserl, E. [Ideen I], "Ideen zu einer reinen Phänomenologie und phänomenologischen Philosophie I: Allgemeine Einfuhrung in die reine Phänomenologie", en Husserliana 3-2, Halbband: Ergänzende Texte, Ed. Karl Schuhmann. La Haya, Netherlands: Martinus Nijhoff [Trad. cast.: Ideas relativas a una fenomenología pura y una filosofía fenomenológica, México, Fondo de la Cultura Económica, 1949, Trad.: José Gaos], 1912-1929. 
Husserl, E. $[D L]$, "Die Lebenswelt. Auslegungen der vorgegebenen Welt und ihrer Konstitution. Texte aus dem Nachlass", en Husserliana 39, Ed. Rochus Sowa. New York: Springer, 2008 [1916-37].

Inverso, H., El mundo entre paréntesis. Una arqueología de las nociones de reducción y corporalidad, Buenos Aires, Prometeo, 2014.

Inverso, H., "Heidegger frente a Husserl en la Introducción a la investigación fenomenológica”, Estudios de Filosofía, N.56 (2017), pp. 49-72. https://doi.org/10.17533/udea.ef.n56a04

Johnson, F., "La exclusión del cuerpo en Sein und Zeit y la negación de una fenomenología del cuerpo en el pensamiento de Heidegger", Pensamiento Vol.72, N.270 (2016), pp. 131-45.

https://doi.org/10.14422/pen.v72.i270.y2016.008

Llorente, J., "Heidegger y el estatuto ontológico del cuerpo. Una confrontación con la fenomenología de la carnalidad de Michel Henry", Ideas $y$ Valores Vol.65, N.162 (2016), pp. 261-289. https://doi.org/10.15446/ ideasyvalores.v65n162.48418

Presas, M., "La experiencia del otro”, Escritos de Filosofía, N.12 (1983), pp. 47-51.

Rabanaque, L., "La corporalidad política como corporalidad ética”, Revista pensamiento político N.7 (2016), pp. 91-110.

Ramírez Cobián, M., "El cuerpo por sí mismo. De la fenomenología del cuerpo a la ontología del ser corporal”, Open insight Vol.8, N.14 (2017), pp. 49-68.

Rodríguez Vergara H., "La conciencia de lo corporal: una visión fenomenológica-cognitiva", Ideas y valores N.142 (2010), pp. 25-47.

Rossi, L., "El problema de la comunidad en Ser y tiempo", en: LythgoeRossi, Ser y tiempo.Singularización y comunidad, Buenos Aires, Biblos, 2016, pp. 103-200.

Rossi, M. y Muñiz, M., "Hermenéuticas de la intersubjetividad en Hegel y Husserl”, Revista Eletrônica Estudos Hegelianos Ano 9, N.16 (2012), pp. 67-93.

Sartre, J.P., El ser y la nada. Ensayo de ontología fenomenológica, Buenos Aires, Losada, 2006 [1943].

Sawicki, M., "Body, Text and Science: the Literacy of Investigative Practices and the Phenomenology of Edith Stein", in Stein's work for Husserl, Dordrecht: Kluwer (1997) pp. 153-65.

Staiti, A., "The Pedagogic Impulse of Husserl's Ways into Transcendental Phenomenology", Graduate Faculty Philosophy Journal, N.33.1 (2012), pp. 39-56. https://doi.org/10.5840/gfpj20123313 
Verano Gamboa, L., "El ser corporal: Dasein y lenguaje en el pensamiento de Martin Heidegger”, Convivium N.29/30 (2016-7), pp. 185-202.

Volpi, F., "Ser y tiempo: semejanzas con la Ética Nicomaquea", Signos filosóficos, Vol. VIII, N.16 (2006), pp. 127-47.

Waldenfels, B., "Fenomenología de la experiencia en Edmund Husserl”, Areté Vol.39, N.2 (2017), pp. 409-26. https://doi.org/10.18800/ arete. 201702.008

Walton, R., "Facetas de la corporalidad en la ética husserliana", Eidos N.21 (2014), pp. 237-59. https://doi.org/10.14482/eidos.21.6492

Zahavi, D., Husserl and Transcendental Intersubjectivity, a Response to the Linguistic-Pragmatic Critique, Behnke, E. (trad.), in Athens: Ohio University Press, 2001. 